\title{
HONESTIDAD MATERIAL. CASTLECRAG 1920-1937
}

MATERIAL HONESTY. CASTLECRAG 1920-1937

Javier Mosquera González

RESUMEN La arquitectura producida a comienzos del siglo XX por la denominada Escuela de Chicago, generó un estilo reconocible más allá de sus fronteras. Tanto es así que muchos de los arquitectos norteamericanos pertenecientes a aquel período han sido considerados como miembros del mismo grupo. Tal es el caso de Marion Mahony Griffin y Walter Burley Griffin, quienes además en su juventud fueron colaboradores de Frank Lloyd Wright junto con el que desarrollaron múltiples proyectos de las Prairie Houses. Sin embargo, una vez comienzan su práctica profesional de manera autónoma en el continente australiano, la distancia establecida entre América y Oceanía les permite evolucionar lo aprendido de sus maestros hacia un lenguaje personal alejado de sus referencias. Aún siendo reconocibles sus orígenes, la arquitectura producida para la comunidad residencial de Castlecrag, muestra unas construcciones singulares, ajenas a lo que en su país natal se estaba produciendo, y que por tanto permite afirmar que no son unos simples discípulos de la Escuela de Chicago, sino unos arquitectos en busca de un lenguaje propio, desde la libertad de quien trabaja en la distancia.

PALABRAS CLAVE Australia; Castlecrag; naturaleza; entorno; Knitlock; Griffin

SUMMARY The architecture produced in the early 20th century by what is known as the Chicago School led to a style that can be found far beyond its borders. Such is the case that many North American architects from that period have been considered members of the same group. They include Marion Mahony Griffin and Walter Burley Griffin, who also collaborated with Frank Lloyd Wright early on, developing several Prairie House projects. However, once they embarked on their own independent professional work on the Australian continent, the distance between America and Oceania allowed them to evolve what they had learnt from their masters towards a more personal language that strayed from their references.

Even with recognizable origins, the architecture produced for the residential community of Castlecrag reflects singular structures quite unlike what was being produced in their home country and which, therefore, proved they were not simply disciples of the Chicago School but rather architects in search of their own language from the freedom of working at a distance.

KEY WORDS Australia, Castlecrag, Nature, Environment, Knitlock, Griffin

Persona de contacto / Corresponding author: jmg@mosqueragonzalez.com. Escuela Técnica Superior de Arquitectura, Universidad Politécnica de Madrid. España 


\section{$\mathrm{M}$}

LA BÚSQUEDA DE UNA IDENTIDAD PROPIA arion Mahony Griffin y Walter Burley Griffin, nacidos a finales del siglo XIX en Chicago (figura 1), comienzan su trayectoria profesional junto a la figura de Frank Lloyd Wright. Gran parte de la literatura disponible sobre estos arquitectos, les relaciona con él llegando a referirse a ellos como sus discípulos ${ }^{1}$. Tras graduarse y trabajar en diversos estudios de arquitectura de Chicago, son contratados como colaboradores de Frank Lloyd Wright, lo que les permitió participar en la mayoría de los proyectos residenciales de las Prairie Houses realizados a las afueras de Chicago. La gran capacidad gráfica como delineante de ella, y los conocimientos y el interés por la arquitectura del paisaje de él, les convirtieron en colaboradores aventajados de Wright. Tras cerca de diez años junto a él, desavenencias económicas les obligan a abandonar el estudio de Oak Park y comenzar su carrera en solitario, dejando atrás una etapa crucial en su formación como arquitectos.

Habiendo realizado algunos proyectos residenciales y estudios urbanísticos en los Estados Unidos deciden participar en el concurso internacional para desarrollar la

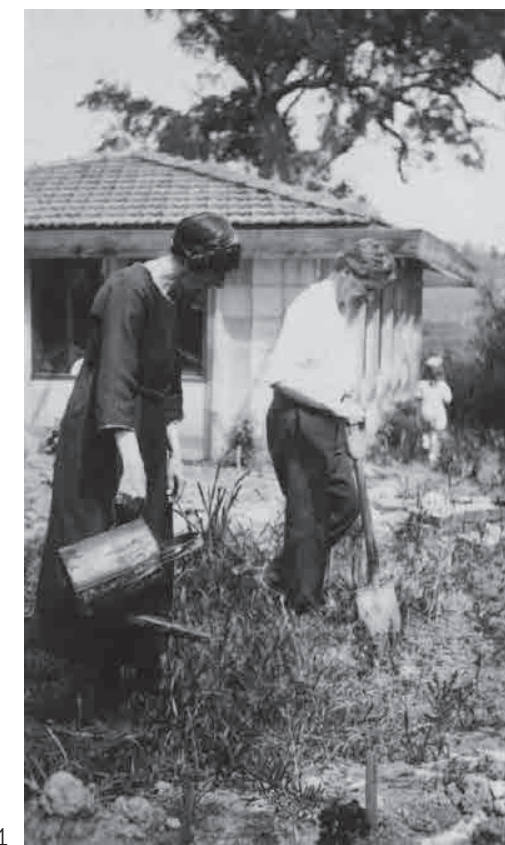

Capital Federal de Australia en Canberra, en gran medida gracias a la voluntad de Marion Mahony Griffin por conseguir su primer gran encargo como arquitectos. Tras resultar vencedores en 1912 y trasladarse a vivir allí, inician el desarrollo de su actividad profesional en el continente australiano adaptándose rápidamente a la nueva realidad física y social en la que se instalan. Su lenguaje arquitectónico puede considerarse como singular y no una mera aplicación de los principios americanos fuera de los Estados Unidos. Una arquitectura en la que se descubren influencias de otras más conocidas pero que se produce al margen de los focos principales de los continentes americano y europeo, y que genera construcciones difícilmente clasificables.

El perfil ecléctico que define el pensamiento de Mahony y Griffin, se debe en gran parte a los múltiples intereses relacionados con cuestiones sociales y políticas que ambos apoyaban. En su juventud sentían una afinidad por personajes que defendían la libertad social y económica, entendida como una búsqueda de la individualidad dentro de un sistema comunitario. Sin embargo, a medida que su personalidad como arquitectos se va 
2. Castlecrag. Plano de situación.

3. Castlecrag. Esquemas. construyendo, su mentalidad se vuelve más compleja al aceptar la existencia de otros factores cercanos al entendimiento de un orden superior de carácter filosófico que busca la comunión entre persona y naturaleza. Pese a todo, la búsqueda de la identidad propia de cada individuo, como elemento que forma parte de un todo superior, entendido éste como comunidad, será una constante en sus vidas.

La individualidad defendida por el pensamiento trascendentalista necesita de la relación bidireccional entre ser humano y entorno natural2 . El arquitecto sólo será capaz de desarrollar una identidad propia, si busca la solución en el hombre. Recuperar la posición central de este frente a la creciente industrialización empuja a arquitectos y demás pensadores a buscar soluciones alternativas al modelo social de la época. Es entonces cuando el término orgánico aparece ligado a la arquitectura, desde la visión de la disciplina en relación con el entorno y de su capacidad de adaptación al mismo. Si bien los trascendentalistas reclamaban la nueva identidad americana, Mahony y Griffin entenderán que este pensamiento puede adquirir un potencial universal capaz de ser desarrollado en cualquier otro continente.

La comunidad de Castlecrag, desarrollada a las afueras de Sídney entre 1920 y 1937, es la obra en la que logran su objetivo de crear un modelo de comunidad ideal, como síntesis construida de su pensamiento global (figura 2). El entorno natural sobre el que se asienta el conjunto de Castlecrag, se caracteriza por su carácter rocoso y escarpado. Siguiendo las curvas de nivel existentes, el proyecto ordena el sistema de viales para vehículos necesarios para absorber el tráfico indispensable, con un trazado sinuoso que bordea las colinas adaptándose al terreno. La parcelación propuesta se adapta a la topografía y permite el acceso a las todas las viviendas desde las vías rodadas principales.

Existe también una red de caminos peatonales secundaria, que conecta diferentes espacios de reunión y ocio al aire libre, y que constituye el verdadero valor añadido de la propuesta con respecto a otras alternativas coetáneas. Será este trazado de caminos y sendas quebradas,

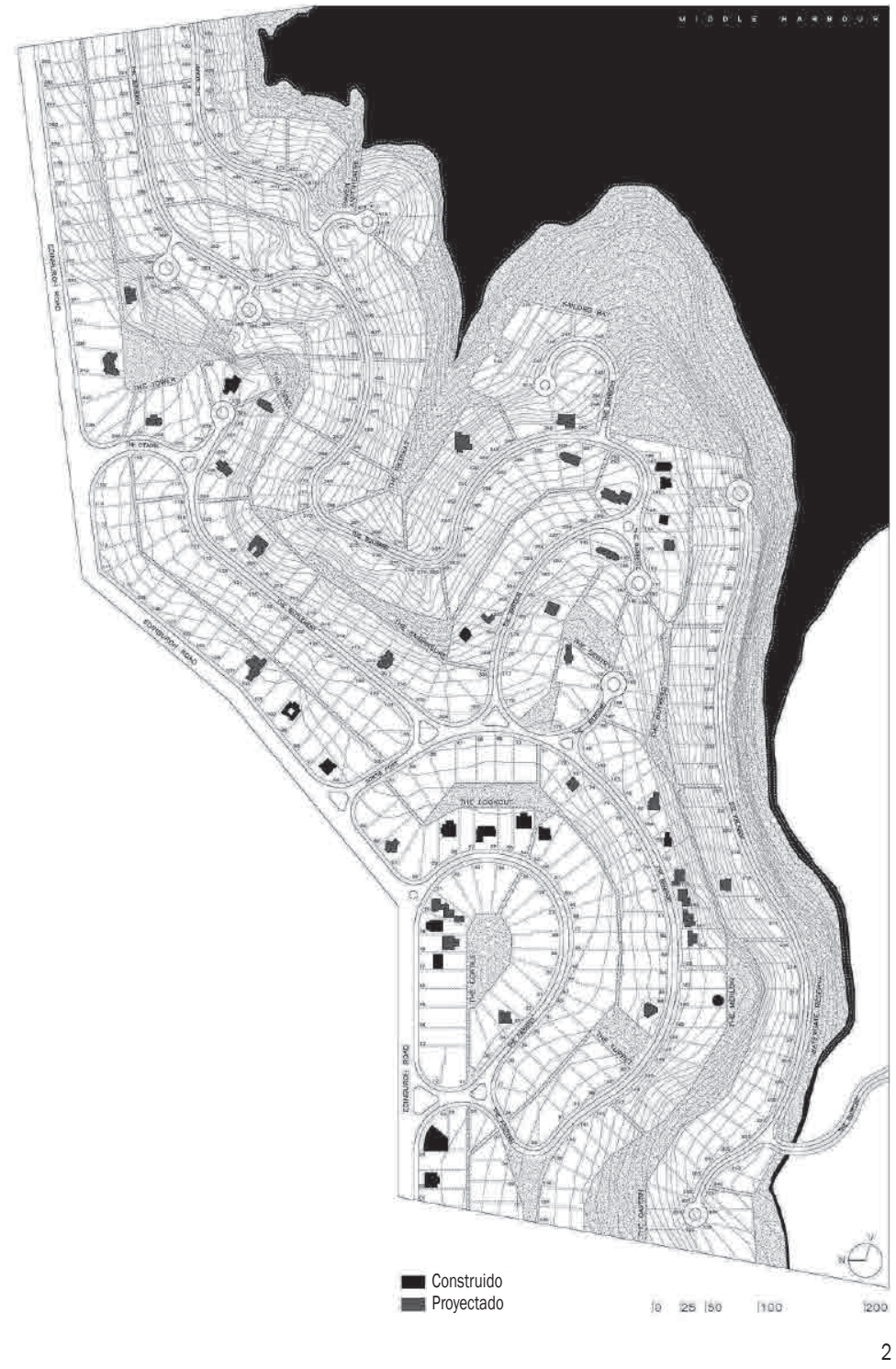


el que dote de significado social al proyecto. Concebida como una pequeña infraestructura organizada de forma que cada parcela pueda acceder directamente a ella sin necesidad de cruzarse con el tráfico rodado, disfrutarán así de espacios comunes en los que compartir actividades culturales y deportivas con el resto de miembros de la comunidad (figura 3).

La ordenación planteada en Castlecrag recoge principios básicos del Pintoresquismo del siglo XIX, al remarcar la importancia de las vistas al mar desde cualquiera de las construcciones propuestas, sin que exista una interferencia entre ellas, así como desde los distintos caminos peatonales que conectan las parcelas entre sí y con el mar. A su vez, la concepción utópica de las comunidades planteadas en Europa a comienzos del siglo XX por Bruno Taut $^{3}$ y la necesidad de la vuelta del hombre a la naturaleza alejado de los centros urbanos industrializados, encontrarán en Castlecrag un modelo construido. Los dilemas derivados de la confrontación entre los modelos productivos urbanos y los rurales, se sustituirán por la reivindicación de un modelo de ocio y convivencia comunitario en relación con la naturaleza y los seres humanos, frente al individualismo promulgado por otras promociones inmobiliarias cercanas a Castlecrag ${ }^{4}$.

Siendo esta su obra más personal y comprometida socialmente, la investigación trata de profundizar en el trabajo de ambos, desde la relación existente entre la naturaleza y sus habitantes, y la manera en la que la construcción es capaz de mostrar con honestidad esta convivencia entre lo existente y lo creado por la mano del hombre.

\section{LA PERTENENCIA AL LUGAR}

El papel del arquitecto en la definición de las nuevas relaciones entre entorno y arquitectura, entre la naturaleza y las personas que en ella habitan, debe reflejarse no solo en el trazado general de la ordenación o en el modelo de comunidad planteado, sino también en la forma en la que estas son construidas. Las acciones que se
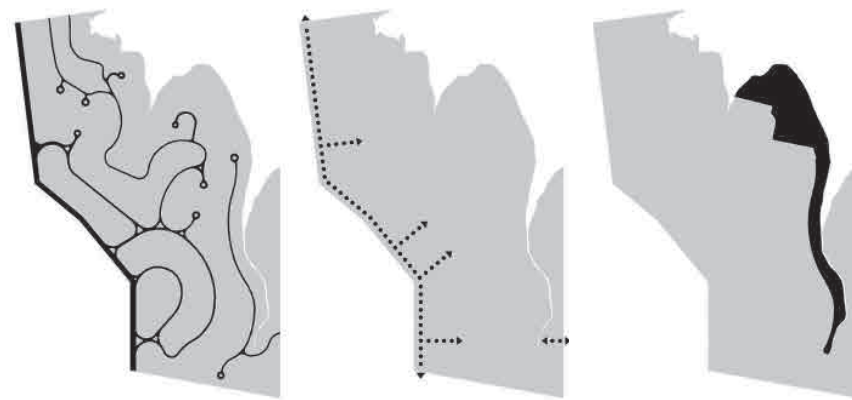

TRÁFICO RODADO ACCESO RODADO

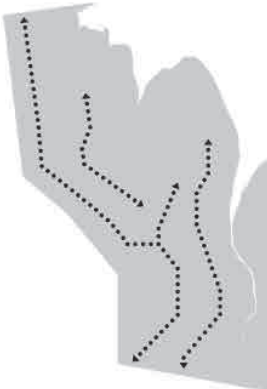

REC. PEATPONALES PRINCIPALES

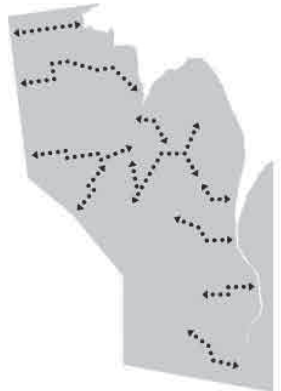

REC. PEATONALES SECUNDARIOS
RECORRIDO PEATONALES

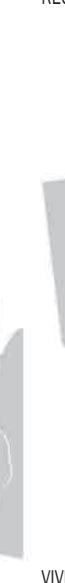

ESPACIOS PÚBLICOS

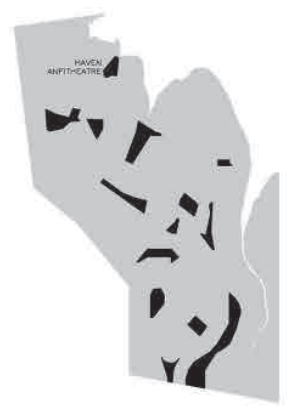

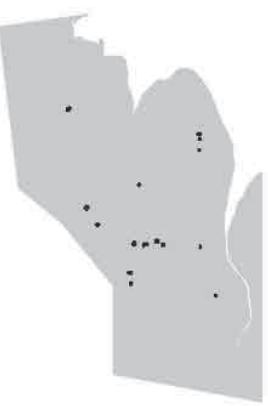

VIVIENDAS CONSTRUIDAS (16)

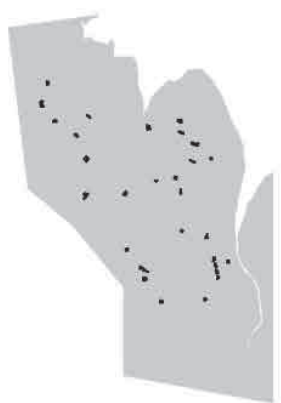

VIVIENDAS PROYECTADAS (27)

3. TAUT, Bruno. Escritos expresionistas 1919-1920. Madrid: El Croquis Editorial, 1997, pp. 256-258.

4. BANERJI, Shiben. "Inhabiting the world: Architecture, Urbanism, and the Global Moral - Politics of Marion Mahony and Walter Burley Griffin", Director: Arindam Dutta. Tesis doctoral. Massachusets Institute of Technology MIT, 2015, pp. 158-162. 
$1920 \ldots$
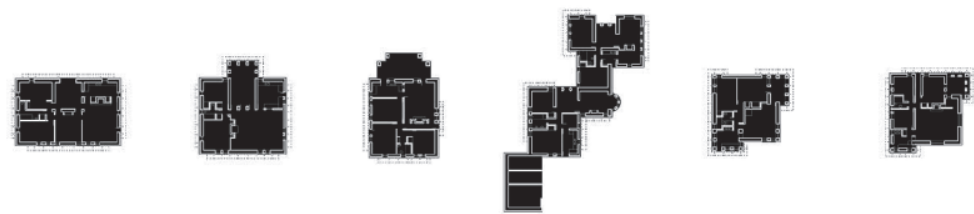

1922
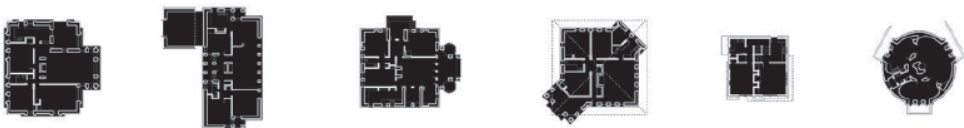

1923

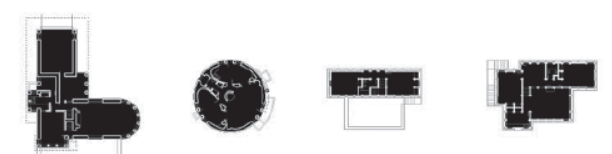

1923
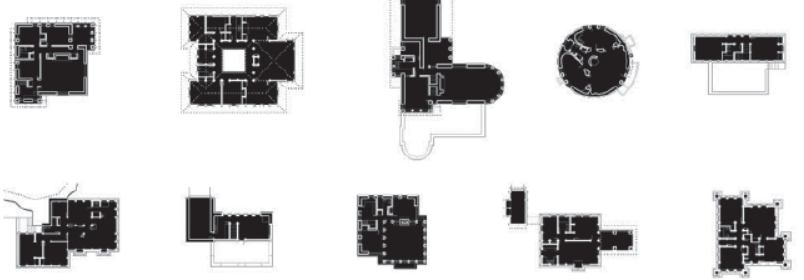
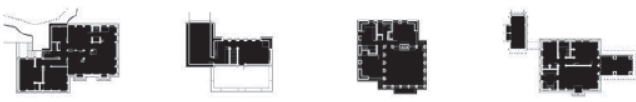

1925
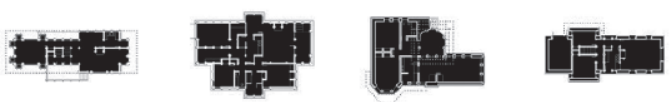

1926
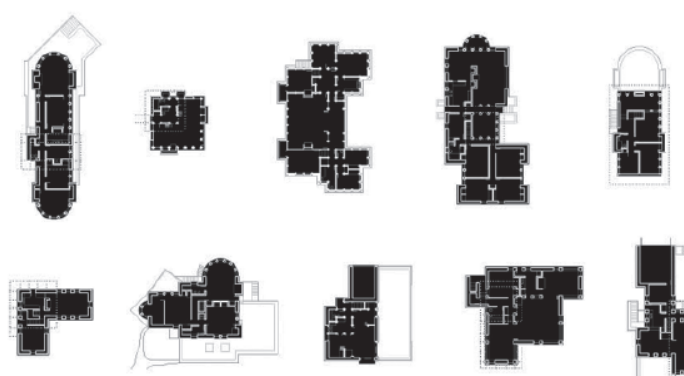
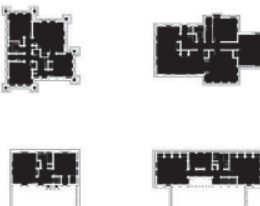

1926
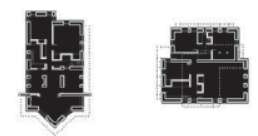

1929

1924

1928
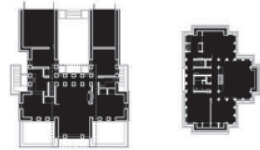

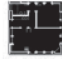

...1937
4. Castlecrag. Relación de viviendas proyectadas y construidas por Marion Mahony Griffin y Walter Burley Griffin entre 1920 y 1937

5. Castlecrag. Casa Johnson.

6. Castlecrag. Casa Wilson. realicen sobre el territorio deberán revelar el potencial inicial del lugar mediante la transformación y manipulación del material a manos del hombre, de forma que el resultado construido sea un reflejo del valor de la naturaleza de la que surge. La construcción no debe considerarse como un hecho aislado, ajeno al lugar en el que se produce, sino que el origen del material y la manera en la que se transforman, han de ser coherentes con la concepción global del proyecto.

El carácter telúrico de muchas de las construcciones realizadas por Henry Hobson Richardson ${ }^{5}$ en los Estados Unidos, pueden ser consideradas como el germen de gran parte de la arquitectura de Mahony y Griffin en el continente australiano. En ambos casos, sus arquitecturas son el resultado de la colocación, unos sobre otros, de elementos pétreos extraídos de la tierra en grandes bloques toscos, la sensación de pesadez refuerza la acción de la gravedad como material constructivo básico del arquitecto para consolidar el volumen en el terreno. La materia disponible para su realización se reconoce como local desde la manipulación consciente del paisaje, esto es, desde la acción artificial sobre la naturaleza, de forma que el resultado final siga perteneciendo al todo, ahora incluyendo de nuevo al hombre. La necesidad de ambos de encontrar una nueva identidad propia, está ligada a la definición de unas construcciones enraizadas al terreno.

Los volúmenes construidos se funden así con el entorno hasta no diferenciarse la roca existente de la construcción artificial. Alejados del lenguaje racionalista y funcional relacionado con el mundo industrial, la arquitectura planteada parece centrarse en cuestiones más primitivas. El hombre, al construir con sus propias manos y con elementos disponibles a su alrededor, se procura a sí mismo un lugar en el que resguardarse. El entorno natural en el 


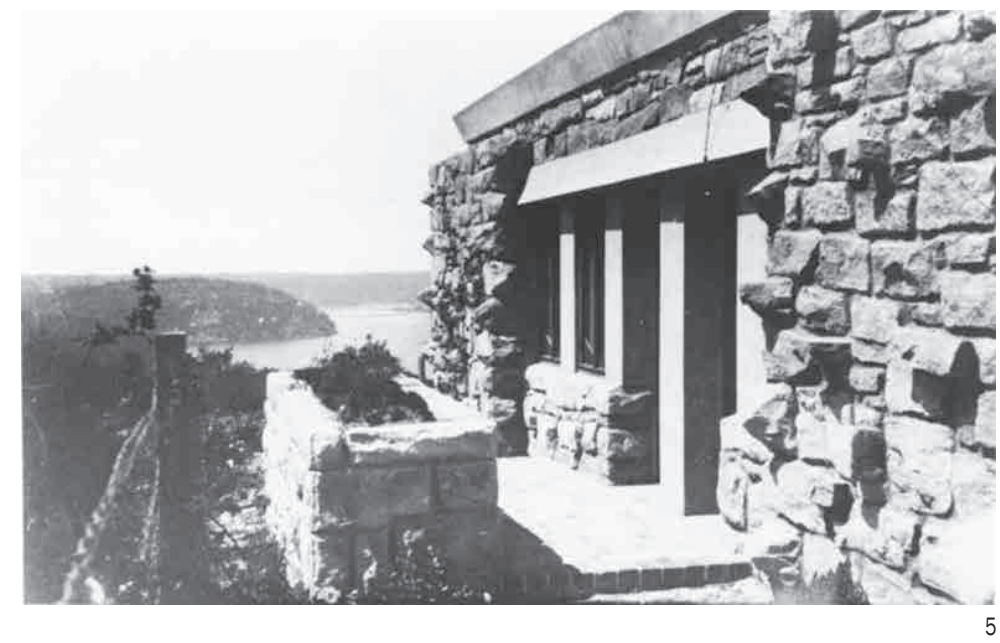

que se asienta la nueva comunidad de Castlecrag a las afueras de Sídney está compuesto por rocas de piedra arenisca, entre las que la vegetación crece generando un lugar sobre el que construir repleto de condicionantes a la hora de proyectar ${ }^{6}$.

Entre los años 1920 y 1937, Mahony y Griffin desarrollan 42 proyectos residenciales unifamiliares para la comunidad de Castlecrag, de los que tan sólo construyen 15. Las tipologías utilizadas varían desde las casas compactas resueltas en una sola planta, hasta ejemplos más complejos en los que el tamaño del encargo les permitía un desarrollo en diferentes niveles, adaptándose a la topografía de una forma más orgánica. Uno de los elementos característicos de todas ellas es la organización en planta de los elementos de servicio, siempre situados en los puntos de acceso, cercanos a las vías peatonales y alejados de las vistas sobre la bahía. El acceder a través de las estancias secundarias y no por las zonas más representativas de la vivienda, supuso un cambio de paradigma entonces que dificultó la aceptación de sus propuestas por parte de la sociedad (figura 4).

Las superficies pétreas de las viviendas, son concebidas como planos en los que existen entrantes y salientes de piezas de distintos tamaños, de tal forma que la incidencia de la luz sobre los mismos acentúa el carácter

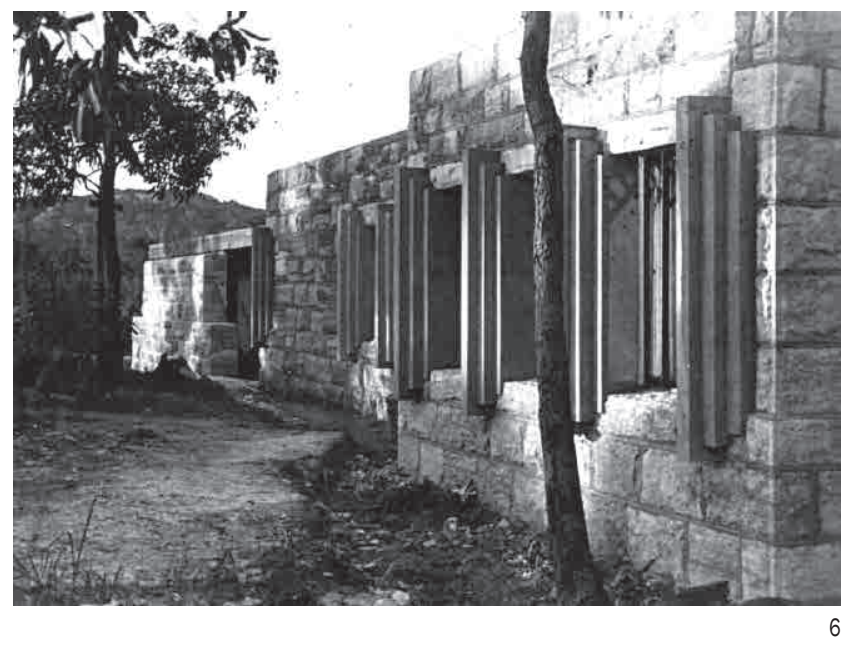

rocoso de las construcciones, destacando así las semejanzas con la topografía y el perfil irregular sobre el que se asientan. De sus fachadas se desprende la relación directa con el lugar, al combinar las piedras que yacen en el terreno, con las talladas expresamente por la mano del hombre, colocadas unas sobre otras como si de un mampuesto se tratara (figura 5).

Existe una aparente exageración constructiva en la manera en la que las viviendas son realizadas. No sólo en el tratamiento de sus muros y el material con el que son construidos, sino en las operaciones que se ejecutan para la apertura de huecos en ellos. La utilización de grandes dinteles y pilastras sobredimensionados, parecen querer significar el esfuerzo estructural que sería necesario aplicar a la hora de abrir huecos y sujetar así el peso de las rocas que sobre ellos se apoyan.

El deseo por enfatizar la condición masiva de las construcciones se traduce en el tratamiento de algunos de los huecos, rodeados de elementos pétreos que se escalonan generando una especie de abocinamiento hacia el exterior. Las sombras arrojadas sobre cada uno de los niveles de estas piezas, servirá para potenciar el efecto de profundidad de estos umbrales, al tiempo que refuerza la condición sustentante de estas partes colocadas entre los huecos (figura 6). 
7. Castlecrag. Planimetría de la Casa Fishwick. 8. Castlecrag. Casa Fishwick.
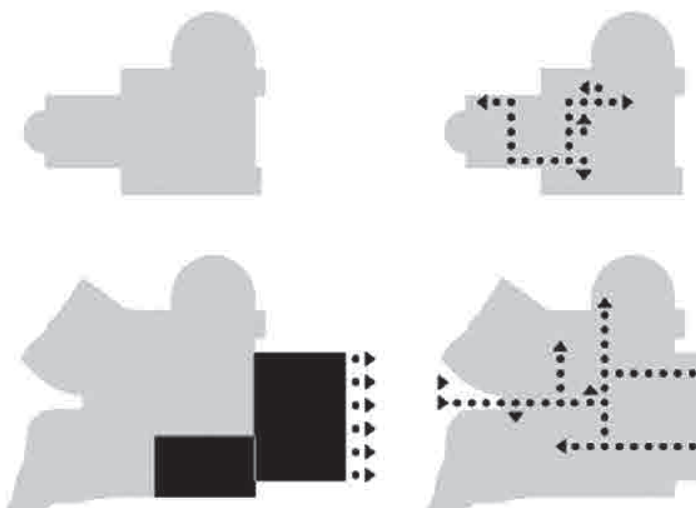

VISTAS
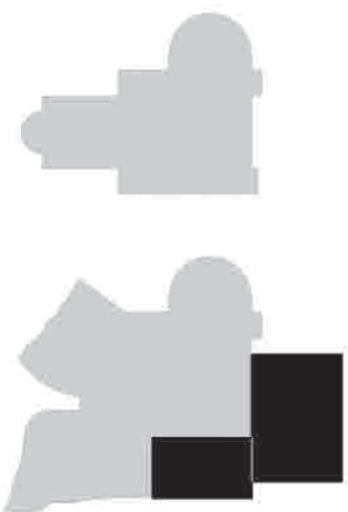

ESP. PRINCIPALES
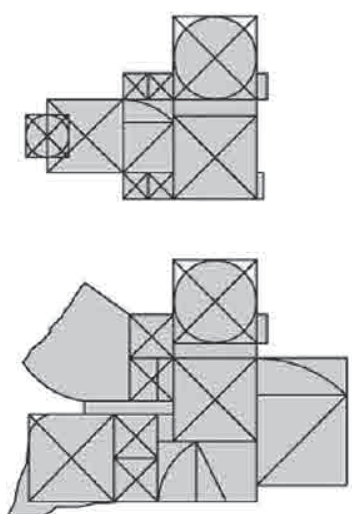

GEOMETRIA

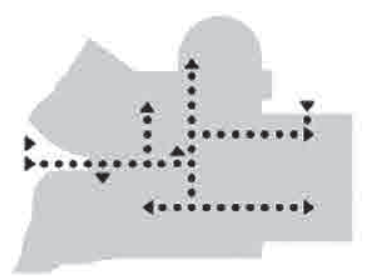

RECORRIDOS
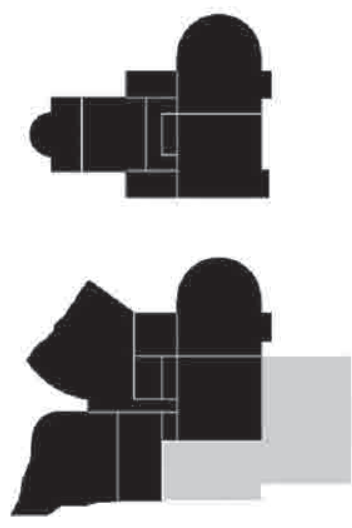

ESP. SECUNDARIOS
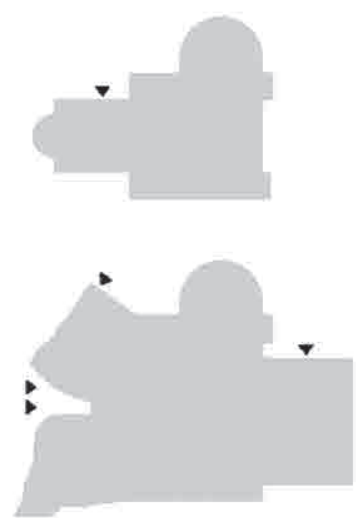

ACCESOS
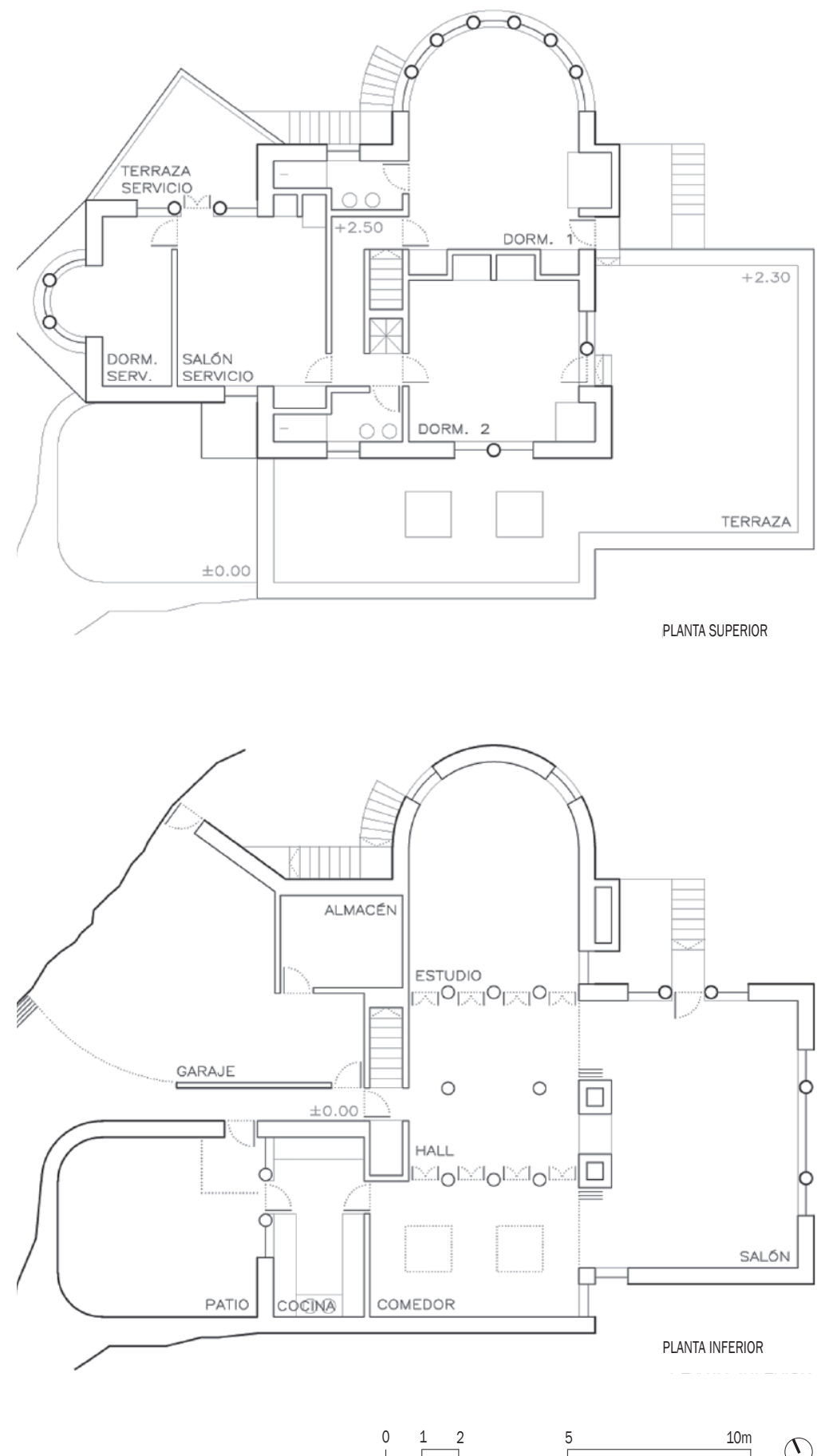


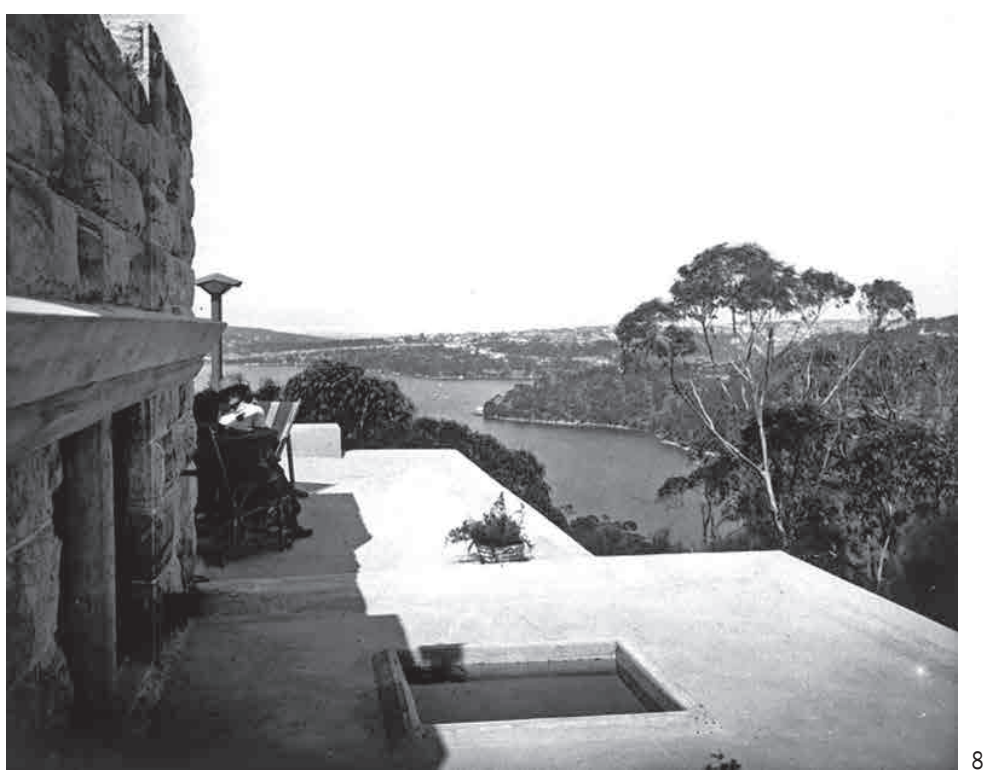

Si las dimensiones de los dinteles de las ventanas resultan en ocasiones desmesuradas, así lo son también las de los pilares que sujetan los elementos de madera que, a modo de pérgola, generan espacios exteriores en sombra. No solo su sección, sino el número utilizado para soportar una estructura ligera de madera, exceden lo estrictamente necesario para cumplir su función. Pueden entenderse estos lugares cubiertos, pero al aire libre, no como una estructura que se añade a posteriori, sino como una estancia más de la casa, que no se cierra con carpinterías ni vidrios. Esta misma solución se utilizará para resolver los elementos situados en las cubiertas de algunos de los proyectos, concebidos como pequeños templos sobre los basamentos de piedra definidos por las viviendas.

En aquellas viviendas enclavadas en lugares en los que la topografía es más pronunciada, la distribución en planta se realiza en dos niveles o más. Aparecen entonces senderos de piedra que serpentean por las laderas escarpadas, adosándose al muro del mismo material que soporta el terreno indicando el camino de acceso a la vivienda. Ocultos tras la vegetación, los recorridos desaparecen, lo que invita a pensar que quien los recorre ya ha llegado a su destino. Los diferentes niveles aterrazados se funden entonces con los volúmenes construidos, de forma que todos ellos parecen contener el empuje de las tierras que quedan a sus espaldas (figura 7). Tan solo la aparición de los huecos significados por las pilastras y las carpinterías, permiten reconocer el volumen masivo como un lugar en cuyo interior se puede habitar.

Las viviendas se configuran de tal manera que los niveles inferiores servirán de terrazas a los niveles superiores. Mediante esta operación, se genera un plano horizontal abstracto, ajeno a las irregularidades del terreno, desde el que contemplar las vistas de la bahía. Sin barandillas de ningún tipo, el paisaje se recortará en el borde del prisma de la vivienda dibujando un contorno geométrico que enmarca la naturaleza sin interponer ningún límite físico entre esta y el residente (figura 8).

Los modelos que servían entonces como referente para la construcción de viviendas en Australia variaban entre el estilo Georgiano, el Shingle Style o la arquitectura realizada en hierro fundido, Iron Lace, heredados de la tradición inglesa y de la arquitectura americana de finales del siglo XIX7. Ajenos de manera voluntaria a estos estilos, Mahony y Griffin propusieron en Castlecrag una alternativa al modelo residencial tradicional. La vida en relación directa con la naturaleza y el respeto que hacia 


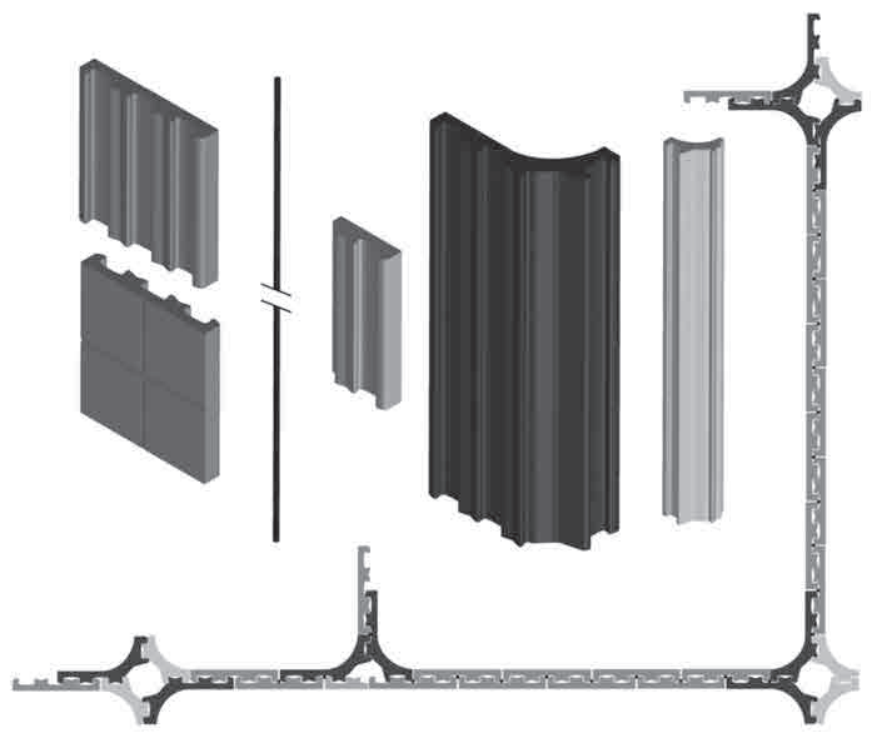

ella mostraban sus vecinos, debían estar implícitos en la forma en la que las viviendas eran construidas.

La combinación de todas estas operaciones da como resultado una arquitectura inquietante, difícilmente clasificable, y a la que en ocasiones podría atribuírsele cierta monstruosidad debido a sus constantes desproporciones. Cercanas en muchas ocasiones a un lenguaje arcaico, sus fachadas remiten al reencuentro del hombre con la naturaleza y las construcciones primitivas realizadas en piedra. Parecen reflejar el proceso de búsqueda de un lenguaje propio, consciente de sus orígenes pero deseoso de producir, desde la imaginación, una realidad alternativa a la conocida.

\section{ARQUITECTURA DEMOCRÁTICA}

Su arquitectura, fue concebida como un bien que debía estar al alcance de todos eliminando las barreras existentes entre diferentes clases sociales. La universalidad por ellos reclamada podía alcanzarse en la construcción me- diante la creación de un sistema capaz de ser exportado a cualquier lugar. Así lo indica Mahony cuando advierte que "si a una casa de bajo coste se le da la belleza e individualidad de una casa de mayor coste la conciencia de la pertenencia a una u otra clase social desaparecerá, ya sea en un pequeño distrito como en un gran país"

En sus palabras aparece implícita la creciente fractura entre las clases sociales como consecuencia de las diferencias de ingresos entre ellas. Se deduce también una crítica al sistema político que favorece estas situaciones, y con el que ambos no se sienten identificados. La creciente deshumanización de la sociedad repercute en la manera que tienen de concebir su arquitectura. Sin embargo, la situación denunciada por ellos no implica que su construcción no se sirva de la técnica para pertenecer al tiempo en el que se produce. Simplemente reclaman un uso diferente de ella.

Conscientes del coste que suponía construir viviendas con bloques de piedra, muchas de las casas de la

8. En referencia a la definición de Democracia enunciada por Louis Sullivan, quién la considera no solo como una forma de gobierno, sino como una fuerza que empodera a cada individuo para encontrar así su verdadera identidad, aún formando parte de un modelo de vida en comunidad. SULLIVAN, Louis Henry. The Public Papers. Editado por Robert Twombly. Chicago: Chicago University Press, 1988, pp. 97-99.

9. GRIFFIN, Marion Mahony. The Magic of America. Chicago: The Art Institute of Chicago, 1949 (2008), pp. 555-560, [consulta: 05-07-2017]. Disponible en: http://www.artic.edu/magicofamerica/index.html.

10. TURNBULL, Jeffrey. En Second International Congress on Construction History 3, The Architecture of Walter Burley Griffin: Concrete applications. Melbourne: Construction History Society, 2006, pp. 3135-3136, [consulta: 10-08-2017]. Disponible en: https://www.arct.cam.ac.uk/Downloads/ichs/vol-3-3133-3154turnbull.pdf 
comunidad de Castlecrag fueron proyectadas con un sistema de construcción prefabricada llamado Knitlock, patentado en 1917 (figura 9). Se trata de un sistema de construcción por segmentos de hormigón armado, desarrollado durante su estancia en Canberra ${ }^{10}$.Si las máquinas resultaban ser el futuro, el hombre debía ser capaz de utilizar sus ventajas para no quedar oculto tras el poder de la producción industrial. Así lo entendieron al crear Knitlock, un sistema capaz de hacer avanzar el proceso constructivo manual tradicional hacia un modelo en consonancia con el desarrollo industrial de entonces.

Griffin, junto con David Charles Jenkins y Malcolm Stewart Moore, desarrollaron la maquinaria necesaria para la elaboración de los elementos de hormigón prefabricado ${ }^{11}$. Cada pieza se fabricaba de manera individual vertiendo hormigón en un molde sobre el que, antes de su secado, vertían arena extraída de las rocas locales. Así, una vez se secaban las piezas, la tonalidad y los reflejos de cada una de ellas se asemejaba a las rocas presentes en el entorno donde se iba a construir la vivienda. Será esta la verdadera relación entre hombre y máquina defendida por ambos. Aquella en la que el primero la utiliza en su beneficio, no para reproducir modelos conocidos, sino para hacer evolucionar la arquitectura en busca de aquella perteneciente a su tiempo y que sea capaz de mantener la esencia del lugar en el que nace.

Las máquinas ideadas servían para fabricar piezas de peso reducido y tamaño compacto, de forma que se pudiesen reducir gastos en la fabricación y en el transporte. Además, la puesta en obra no necesitaba de formación cualificada y cada módulo podía ser colocado por un operario de forma individual, acelerando así el tiempo de montaje.

El sistema estaba compuesto por las vértebras, en las esquinas, y las teselas cuadradas en las superficies continuas. Cada una de ellas dispone de una cara lisa, acabada en hormigón, y una cara interior conformada con una serie de acanaladuras verticales. El sistema constructivo se ejecuta mediante la unión en su parte con las acanaladuras, de forma que encajen unas con otras, permitiendo la introducción de redondos de acero allí donde fuese necesario. En la unión de unas con otras se coloca una capa bituminosa que impermeabiliza el interior, al tiempo que las acanaladuras resultantes facilitan el paso del aire así como de instalaciones eléctricas y de gas. El espesor total del cerramiento propuesto es de unos $7,5 \mathrm{~cm}$, resolviendo a la vez estructura, fachada y acabados interiores.

El aspecto masivo y pesado de la construcción con bloques de piedra deja paso a unas edificaciones en las que las aristas propias del Knitlock aumentan la sensación de esbeltez y ligereza de la solución. Es por este motivo que muchas de las viviendas resueltas únicamente con este sistema constructivo de mayor compacidad, realizadas entre 1924 y 1928, resultan ser más pequeñas en el conjunto de Castlecrag (figura 10). En la composición de las plantas se observa la utilización del módulo de los paneles prefabricados como generador de las distribuciones interiores, lo que en ocasiones da lugar a esquemas rígidos que impiden una relación más intensa con su entorno natural inmediato.

El sistema prefabricado ha de entenderse como una respuesta desde la técnica al problema de la construcción pesada en relación con el entorno natural, que servirá como complemento ideal al uso de bloques de piedra apilados. Al exterior las viviendas tratarán de configurarse con este tipo de bloques, ya sea en los muros perimetrales, en los zócalos o en ambos. El interior resolverá los requisitos estructurales y de organización funcional gracias al empleo del Knitlock. Los huecos de ventanas y puertas se convertirán en superficies de mayor tamaño sobre las que se realizarán composiciones diversas mediante el uso de carpinterías de madera.

La honestidad del material, esto es, aquel uso que muestre su naturaleza sin tratar de imitar a ningún otro, es algo que les preocupaba en sus proyectos en el continente australiano. Precisamente por este motivo ambos aseguran que su sistema de construcción prefabricada era el más adecuado, ya que todas las decisiones que se habían tomado para su diseño respondían a necesidades reales, a problemas a los que se daba solución con este producto. "Todos los materiales de la naturaleza son bellos, por tanto las estructuras y nuevas construcciones 


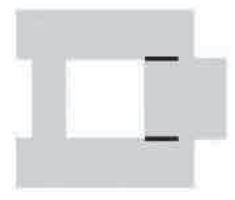

DIVISIONES MOVILES

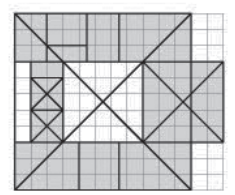

GEOMETRIA

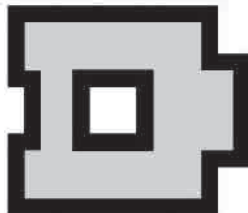

ESPACIOS SOMBRA

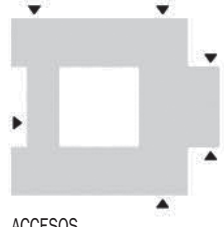

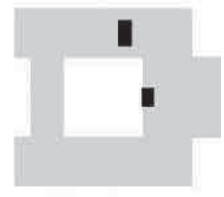

CHIMENEAS

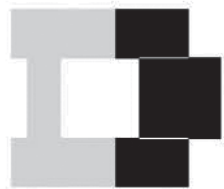

ESPACIOS PRINCIPALES

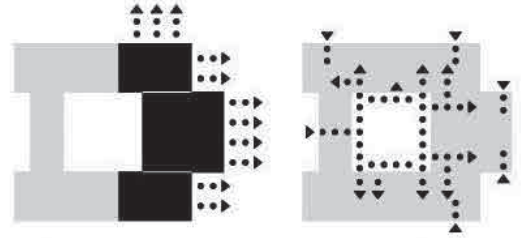

VISTAS

RECORRIDOS

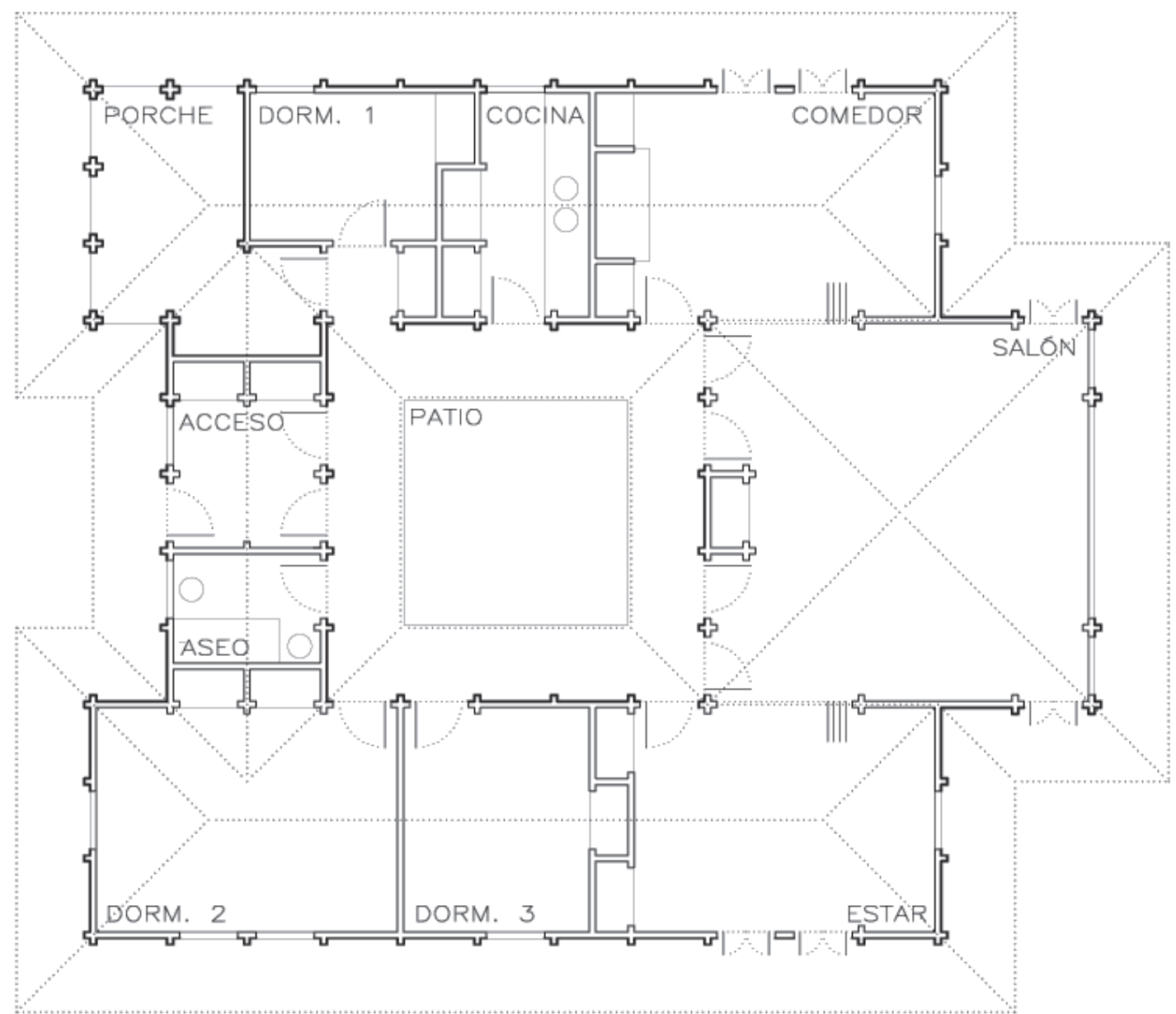


serán bellas si se usan con franqueza y se tratan según sus características naturales"12.

La utilización de áridos locales en la mezcla de hormigón, ha de entenderse como una voluntad por pertenecer al lugar desde la manipulación de la materia, y no como un ejercicio de imitación de los bloques de piedra extraídos directamente del terreno. Así, abogan por una honestidad capaz de dignificar no sólo al material utilizado sino a quien lo usa. Hormigón y piedra son los materiales estructurales por excelencia para ambos, por lo que su sistema de construcción prefabricada es una combinación de estos.

"La arquitectura debe perseguir la construcción de casas "naturales", verdaderas e íntegras, de forma que el hombre sea una parte más de la nueva estructura orgánica que significa una evolución de la existente"13. La casa "natural" será aquella que como resultado de una transformación a manos del hombre de los recursos disponibles, consiga establecerse en el conjunto de los elementos naturales existentes, de una forma honesta. Será considerada como tal, si tras su concepción y materialización, resulta coherente como parte integrante de un organismo vivo previo a ella como es la naturaleza. Así, la nueva construcción servirá al hombre para ser consciente de su entorno, al tiempo que este formará parte del hombre en su nueva vida.

Del estudio de los sistemas constructivos empleados en Castlecrag, ya sea mediante la construcción con bloques de piedra, el uso de piezas prefabricadas, o una combinación de ambos, se descubre una voluntad de los arquitectos por acercarse a la naturaleza desde la técnica. Surge entonces la confrontación entre el idealismo de quien trata de desarrollar un modelo constructivo ejemplar en su relación con el entorno, y el pragmatismo que les hace tener presentes otros factores económicos que soporten su modelo inmobiliario propuesto. Esta contradicción, tan propia de la cultura anglosajona, les permite plantear un sistema social innovador en el que la honestidad material en la construcción, enfatiza la idea principal de la vuelta consciente del hombre a la naturaleza, para vivir en comunidad; recuperando así las enseñanzas de los que han de considerarse como sus primeros maestros, los trascendentalistas americanos.

\section{HACIA LO UNIVERSAL DESDE LO LOCAL}

Más allá del aspecto formal de las construcciones planteadas en Castlecrag, el interés de estas reside en la coherencia existente entre el pensamiento que origina su arquitectura y la materialización del mismo. Deben entenderse como gestos instintivos en los que la búsqueda de la confirmación de una idea prima sobre la voluntad de concretarla desde un estilo y lenguaje reconocibles. Se trata de construcciones ligadas al terreno, surgidas desde la necesidad de denunciar un olvido de la naturaleza. La manipulación de esta se realizará en términos que consigan ponerla en valor, recordando su origen, pero generando una dualidad entre hombre y entorno derivada de su modificación respetuosa. Solo así el ser humano será parte del todo y el todo será parte del hombre.

No se trata de un proyecto que deba ser estudiado como una obra icónica en la historia de la arquitectura, atendiendo únicamente al objeto construido. Sin embargo, su verdadero valor reside en la capacidad de condensación de muchas de las ideas que, en un período de ebullición intelectual como fueron las tres primeras décadas del siglo XX, recorrían el mundo, en ocasiones de forma paralela. Se trata de un ejercicio de síntesis planteado en un país como Australia, alejado de los continentes europeo y americano, pero que les sirve como lugar de trabajo neutral, ávido por definir su propia identidad como pioneros en una tierra por descubrir.

Su proceso de búsqueda personal se basa en una revisión de todas aquellas influencias recibidas, siendo conscientes de ellas, pero tratando de establecer una distancia desde la que experimentar alternativas personales y poder definir así un lenguaje propio. E comenzar de nuevo en un continente sin referentes locales de arquitectura contemporánea y la libertad para reinterpretar lo aprendido, les permitirá descubrir así su verdadera identidad. Deseosos de convertir en realidad 
sus ideales, deciden pasar a la acción, y guiados por la intuición y la razón, proyectan su obra. En su momento de madurez como arquitectos, realizan una arquitectura con errores, con equivocaciones, torpe incluso desde la ingenuidad, pero con determinación. La coherencia de su pensamiento suple las carencias de un lenguaje incipiente, deseoso de encontrar una alternativa a lo conocido y que, frente a la posición pasiva de otros, les empuja a convertirlo en una realidad construida.
Dispuestos a aceptar el error, apuestan por trazar un camino, tortuoso en ocasiones, que pretende llegar a la universalidad de la arquitectura desde el valor de lo local. Una mirada detenida sobre la figura de Marion Mahony Griffin y Walter Burley Griffin desvela, más allá de la arquitectura propuesta en el proyecto de Castlecrag, una actitud, una forma de activismo, coherente con unos ideales que perseguían tratando de mejorar el mundo en el que vivían. 
PROYECTO, PROGRESO, ARQUITECTURA

41

\section{Bibliografía citada:}

BANERJI, Shiben. "Inhabiting the world: Architecture, Urbanism, and the Global Moral - Politics of Marion Mahony and Walter Burley Griffin". Director: Arindam Dutta. Tesis doctoral. Massachusets Institute of Technology MIT, 2015.

BIRRELL, James. Walter Burley Griffin. Brisbane: University of Queensland Press, 1964.

BOYD, Robin. Australia's home: its origins, builders and occupiers. Melbourne: Melbourne University Press, 1991.

EMERSON, Ralph Waldo. Nature. Boston: James Munroe and Company, 1836.

GRIFFIN, Dustin. The writtings of Walter Burley Griffin. Nueva York: Cambridge University Press, 2008.

GRIFFIN, Marion Mahony. The Magic of America [en línea]. Chicago: The Art Institute of Chicago, 1949 (2008). [consulta: 05-07-2017] Disponible en: http://www.artic.edu/magicofamerica/index.html.

JOHNSON, Donald Leslie. The Architecture of Walter Burley Griffin. South Melbourne: Macmillan Company of Australia, 1977.

SULLIVAN, Louis Henry. The Public Papers. Chicago: Editado por Robert Twombly. Chicago: Chicago University Press, 1988.

TAUT, Bruno. 1997. Escritos expresionistas 1919-1920. Madrid: El Croquis Editorial, 1997.

TURNBULL, Jeffrey. 2006. The Architecture of Walter Burley Griffin: Concrete Applications. En Second International Congress on Construction History, 3: 3133 - 3151. Londres, 2006

ULARGUI AGURRUZA, Jesús. De Richardson a Sullivan. Un nuevo espacio social. Barcelona: Fundación Caja de Arquitectos, 2007.

WALKER, Meredith, KABOS, Adrienne, y WEIRICK, James. Building for Nature. Walter Burley Griffin and Castlecrag. New South Wales: Walter Burley Griffin Society, 1994.

Javier Mosquera González (Madrid, 1983). Arquitecto por la ETSAM UPM 2007, y Máster en Proyectos Arquitectónicos Avanzados por la ETSAM UPM 2017. Forma parte del Proyecto de Innovación Educativa de la Universidad Politécnica de Madrid "El juego de la Ciudad" (ETSAM UPM 2017) dentro de la unidad docente Grupo4, perteneciente al Departamento de Proyectos Arquitectónicos de la ETSAM, en la que desempeña labores de apoyo desde 2013. Entre sus publicaciones destacan "Trazados y trazas. Utopías redefinidas" (Foro de investigación en arquitectura. Arquitectura y sociedad. Crisis y utopía. México, 2017) y "Actionscape" (UIA 2017 Seoul World Architects congress). Doctorando en el programa de Proyectos Arquitectónicos Avanzados de la ETSAM UPM, con la tesis, "La búsqueda de una identidad propia. Una práctica global al margen. Marion Mahony Griffin y Walter Burley Griffin. 1912-1937". 
HONESTIDAD MATERIAL. CASTLECRAG 1920-1937

MATERIAL HONESTY. CASTLECRAG 1920-1937

Javier Mosquera González

p.29 THE SEARCH FOR THEIR OWN IDENTITY

Marion Mahony Griffin and Walter Burley Griffin, born at the end of the 19th century in Chicago (figure 1) began their professional careers alongside Frank Lloyd Wright. Much of the literature available on these architects links them to him, evening referring to them as his disciples. ${ }^{1}$ After graduating and working at different architecture firms in Chicago, they were contracted as collaborators of Frank Lloyd Wright, which allowed them to participate in most of the Prairie Houses residential projects in the Chicago suburbs. Marion's immense graphic capabilities as a draftswoman and Walter's knowledge and interest in landscape architecture led them to becoming outstanding collaborators for Wright. After nearly ten years at his side, economic disagreements forced them to leave the Oak Park studio and begin their own individual careers, leaving behind a crucial stage in their training as architects.

Having worked on some residential projects and urban development studies in the United States, they decided to bid for an international tender to develop Australia's capital city of Canberra, largely due to Marion Mahony Griffin's desire to secure their first major commission as architects. After being granted the tender in 1912 and moving there, they began developing their careers on the Australian continent, quickly adapting to the new physical and social reality of the place where they had settled. Their architectural language can be considered unique and not just a mere application of American principles outside the United States. It is an architecture that reflects influences of other more well-known styles yet produced outside the main focal points of the American and European continents and which generated structures quite difficult to categorize.

The eclectic profile that defines Mahony and Griffin's thought is largely due to their many interests relating to the social and political issues they both supported. In their youth, they identified with people who defended social and economic freedom, understood as a search for individualism within a community system. However, as their personalities

p.30 as architects developed, their mentalities became more complex upon accepting the existence of other factors close to an understanding of a higher philosophical order seeking communion between people and nature. In spite of all this, the search for each individual's own identity as an element that is a part of a higher whole, where this is understood as community, would become a constant in their lives.

The individualism defended by transcendentalist thought requires a two-way relationship between human beings and nature. ${ }^{2}$ Architects are only capable of developing their own identity if they find the solution in man. Recovering man's central position in spite of increasing industrialization pushed architects and other thinkers to find alternative solutions to the social model of that time. That was when the term "organic" began to be connected to architecture, from a view of the field in relation to the environment and its capacity to adapt to it. While transcendentalists were clamoring for the new American identity, Mahony and Griffin understood that this idea could acquire universal potential capable of being developed on any other continent.

The community of Castlecrag, developed on the outskirts of Sydney between 1920 and 1937, is where they achieved their goal of creating an ideal community model as a synthesis of their global thinking (figure 2). The natural environment where Castlecrag sits is characterized by its rocky, craggy terrain. Following the existing level curves, the project designed a system of roads needed to absorb essential traffic with a sinuous line that skirts the hills, thereby adapting to the terrain. The plot division proposed adapts to the topography and allows access to all homes from the main roads.

There is also a secondary system of pedestrian paths that connects different open-air gathering and leisure areas which brings real added value to the proposal with respect to other contemporary alternatives. This outline of broken

p.31 paths and trails is what would make the project socially significant. Designed as a small infrastructure organized in such manner so that each plot could be directly accessed without crossing any vehicle traffic, it would feature common areas to share cultural and sports activities with the other members of the community (figure 3).

The planning of Castlecrag reflects some basic principles of 19th century Picturesque in the emphasis of the importance of ocean views from any of the structures proposed without any interference among them as well as from the various pedestrian paths connecting the plots together and to the sea. At the same time, the utopian concept of community emerging in Europe in the early 20th century with Bruno Taut ${ }^{3}$ and the need to bring man back to nature, far from the industrialized urban centers, would find a built model in the form of Castlecrag. The dilemmas arising from the confrontation between the urban and rural production models would be replaced by claims for a model of community leisure and co-existence in relation to nature and human beings versus the individualism promulgated by other real estate developments near Castlecrag. ${ }^{4}$

Given that this is their most personal and socially-committed work, the research attempts to delve into their work from the relationship that exists between nature and the inhabitants and the way in which a structure is capable of honestly reflecting this balance between what exists and what is created by man.

\section{A SENSE OF BELONGING}

The role of an architect in defining new relationships between the environment and architecture and between nature and the people who inhabit it must not only be reflected in the general organizational lines or the community model 
suggested, but also in the way in which they are built. The action taken on a territory must reveal the initial potential of the site through the transformation and manipulation of the location at the hands of man in such manner that the built result is a reflection of the value of the nature from which it arises. Construction should not be considered an isolated event, entirely unrelated to the location where it occurs. Rather, the origin of the materials and the way in which they are transformed should be coherent with the overall project concept.

The tellurian nature of many of the structures designed by Henry Hobson Richardson ${ }^{5}$ in the United States may be considered as the seed of much of the architecture of Mahony and Griffin in Australia. In both cases, their architecture styles are the result of arranging certain rocky elements extracted from the earth in large crude blocks over another where the sense of heaviness reinforces the action of gravity as the architect's basic construction material to consolidate the volume on the terrain. The material available for its completion is recognized as local from the conscious handling of the landscape; in other words, from the artificial action on nature, in such manner that the final result continues to belong to the whole yet now including man. The need for both to find their own new identity was linked to the definition of structures deeply rooted in the land.

The built volumes thus blend in with the environment to such extent that the existing rock cannot be differentiated from the artificial structure. Far from the rationalist and functional language related to the industrial world, the architectural style created seems to focus on more primitive matters. Upon building with his own hands and with elements available around him, man secures a place to shelter himself. The natural environment where the new community of Castlecrag sits on the outskirts of Sydney is comprised of sandstone rocks among which the vegetation p. grows, producing a place where construction must be planned with reference to a number of conditioning factors. ${ }^{6}$

Between 1920 and 1937, Mahony and Griffin developed plans for 42 single-family residential projects for the community of Castlecrag, of which only 15 of were ever built. The types used ranged from compact one-story homes to more complex examples where the size of the job allowed them to develop various levels, adapting to the topography in a more organic manner. One of the characteristic elements of all of them is the plan-view organization of the service elements, always located at entrances near pedestrian paths at a remove from the views of the bay. Entering through secondary rooms and not the most representative areas of the home was a change in paradigm at the time that made it difficult for society to accept their proposals (figure 4).

The rocky surfaces of the homes are designed flat with incoming and outgoing parts of different sizes in such way that the way in which light falls on them emphasizes the rocky nature of the structures, thus highlighting the similarities with the topography and irregular profile over which they stand. The facades reflect a direct relationship with the place by combining the rocks already on the land with those specifically carved by man and arranged one over the other much like a stone wall (figure 5).

There is obvious constructive exaggeration in the way in which the homes were built. Not only in the treatment of the walls and the material with which they were built, but also the work done to create gaps in them. The use of large lintels and oversized pilasters seem to suggest the structural effort that would be needed when opening up the gaps and securing the weight of the rocks supported thereon.

The desire to emphasize the sheer mass of the structures can be seen in how some of the gaps are treated, surrounded by rocky elements staggered to create a sort of flaring on the outside. The shadows over each of the levels of these pieces boost the effect of the depth of the thresholds all while reinforcing the supporting function of these parts placed between the gaps (figure 6).

If the dimensions of the window lintels are at times excessive, so are the pillars supporting the wooden elements which, much like a pergola, create shady outdoor areas. Not just in terms of their sectioning but also the number used to support a light wooden structure, exceeding what is strictly necessary on a functional basis. These covered yet open-air areas may be understood not as a structure added on at a later time but rather as just another room in the house that is not closed with carpentry or glass. This same solution would be used for the elements on the roofs of some of the projects, designed as small temples over the stone foundations defined by the houses.

In the homes enclaved in areas where the topography is more pronounced, the floor distribution is on two or more levels. Stone paths emerge, winding along the craggy slopes next to a wall of the same material that supports the land, indicating the road giving access to the home. The routes disappear amid the vegetation, which leads one to think the person who takes them has already reached their destination. The various terraced levels blend in with the built volumes in such way that all of them seem to contain the push of the land behind (figure 7). Only the appearance of the significant gaps in the pillars and carpentry work make it possible to recognize the massive volume as a place where someone may live.

The homes are designed in such way that the lower levels are used as terraces for the upper levels. This creates an abstract horizontal plane in contrast to the irregularities of the land to take in the views of the bay. Without any railings of any kind, the landscape is cut off on the edge of the prism of the home, creating a geometric contour that frames the surrounding nature without forming any physical boundary between nature and the resident (figure 8).

The models that were used as a reference for homebuilding in Australia varied between Georgian, Shingle Style, Cast Iron and Iron Lace, which were inherited from late 19th century English traditions and American architecture. ${ }^{7}$ 
Voluntarily disconnected from these styles, Mahony and Griffin proposed an alternative to the traditional residential model for Castlecrag. Life in direct contact with nature and with the respect for nature shown by the locals had to be implicit in the homes being built.

The combinations of all these activities resulted in architecture that was troubling and difficult to classify, at times attributed certain monstrosity due to the constant disproportions. Often close to an archaic language, the facades refer to an encounter between man and nature and primitive stone-made structures. They seem to reflect the process of searching for one's own language, aware of one's origins yet yearning to produce an imaginative alternative to the known reality.

\section{DEMOCRATIC ARCHITECTURE ${ }^{8}$}

Their architecture was conceived as an asset that should be within everyone's reach, eliminating the existing barriers between the different social classes. The universality they demanded could be seen in construction through a system that could be exported anywhere. So stated Mahony when he warned that, "if a low-cost house is given the beauty and individuality of a home of a higher cost, the awareness of belonging to one social class or another will disappear in small districts as well as large countries."

His words seem to imply the growing fracture among social classes as a result of the differences in income between them. A criticism of the political system that fostered these situations, a system which neither identified with, can also be deduced. The increasing dehumanization of society is reflected in the way in which they conceived their architecture. However, the situation they denounced does not imply that the construction does not use technique to belong to the time in which it was produced. They were simply calling for a different use.

Aware of the cost of building homes with stone blocks, many of the homes in the community of Castlecrag were

p.37 planned with a prefabricated construction system known as Knitlock, which was patented in 1917 (figure 9). It is a reinforced concrete segment-based construction system that was developed when they were living in Canberra. ${ }^{10}$ If machines were the future, man had to be able to use its benefits so as not to become lost behind the power of industrial production. That was the understanding behind the creation of Knitlock, a system that was capable of moving the traditional manual construction process towards a model that was more in line with the industrial development of that period.

Griffin, along with David Charles Jenkins and Malcolm Stewart Moore, developed the machinery necessary to produce the prefabricated concrete elements. ${ }^{11}$ Each piece is produced individually by pouring concrete into a mold over which sand, extracted from local rocks, was poured before setting. Thus, the tone and reflections of each of the pieces looked similar to the rocks in the environment where the home was built after setting. This would be the real relationship between man and machine, as defended by both. It is a relationship where the first uses it to his own benefit, not to reproduce known models, but rather to force architecture to evolve in search of that which belonged to the times and was capable of maintaining the essence of the location where it was born.

The machines designed were used to manufacture low-weight and small-size pieces to reduce production and transportation costs. Moreover, installing them did not require any training and each module could be positioned by one worker individually, thereby accelerating the assembly time.

The system was comprised of vertebrae on the corners and squared tesserae on the continuous surfaces. Each featured a smooth side, finished in concrete, and an inner side formed by a series of vertical grooves. The construction system involved joining the grooves to fit one into another, inserting steel caps wherever necessary. When joining one to another, a bituminous layer would be poured to weatherproof the inside whereas the resulting grooves would facilitate the passage of air as well as electrical and gas installations. The total thickness of the foundation proposed was some $7.5 \mathrm{~cm}$, which resolved the structure, facade and inner finishes all at the same time.

The massive and heavy look of construction with stone blocks gives way to buildings where the very corners of the Knitlock increase the sensation of thinness and lightness in the solution. It was for this reason that many of the homes built only using this more compact construction system, between 1924 and 1928, are generally smaller than others in Castlecrag (figure 10). The floor plans reflect the use of the prefabricated panel modules to create interior distributions which at times gave rise to rigid outlines preventing a more intense relationship with the immediate natural environment.

The prefabricated system must be understood as a technical response to the problem of heavy construction in relation to the natural environment as the ideal complement to the use of piled stone blocks. Attempts were made to create the outside of the homes with this type of block either in perimeter walls, the base, or both. The interior structural and functional organization requirements would be met using Knitlock. The gaps for the windows and doors became larger surfaces for varying composition using wooden carpentry.

The honesty of the material, in other words, the use reflecting their nature without trying to imitate any other was of concern in their projects on the Australian continent. It was precisely for this reason that they both claimed that their prefabricated construction system was the most appropriate as all decisions that had to be taken for the design met real needs and problems that could be resolved with this product. "All the materials in nature are beautiful; therefore, structures p.39 and new construction will be beautiful if they are used frankly and are treated based on their natural characteristics. "12

The use of local aggregate in the concrete mixes must be understood as a desire to belong to the place through the material handling and not as a means to imitate the stone blocks extracted directly from the land. This was how they advocated honesty capable of dignifying not only the material used but also the person using it. Concrete and stone were the structural materials par excellence for both, meaning their prefabricated construction system was a combination of the two. 
"Architecture must pursue the construction of "natural", real and integral homes where man is just another part of

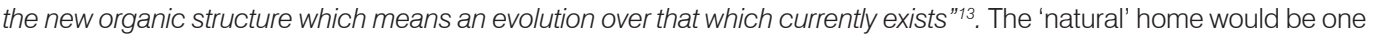
that could be erected among the existing natural elements in an honest manner through the manual transformation by man of the available resources. It would be considered as such if, after its design and materialization, it was coherent as an integrating part of the living organism that existed first, which would be nature. Thus, the new construction would help man be more aware of the environment all while becoming a part of man in his new life.

A study of the construction systems used in Castlecrag, whether through construction with stone blocks, the use of prefabricated parts or a combination of the two, reflect the architects' will to become closer to nature through technique. And thus, there arose a confrontation between the idealism of who attempted to develop an exemplary construction model in relation to the environment and the pragmatism involved when considering other economic factors that supported the proposed real estate model. This contradiction, which is embedded in Anglo Saxon culture, allowed them to contemplate an innovative social system where the material honesty of construction would emphasize the main idea of man's conscious return to nature to living as a community, thereby recovering the teachings that must be considered as their earliest influences - the American transcendentalists.

\section{FROM LOCAL TO UNIVERSAL}

Beyond the formal look of the structures planned in Castlecrag, the interest thereof lies in the coherence between the thinking that originated the architecture and the materialization thereof. They must be understood as instinctive gestures where the search for confirmation of an idea takes precedence over the will to establish it from recognizable style and language. They are structures connected to the land which arise from the need to denounce the abandonment of nature. Nature is handled in a way that values it, recalling its origins, yet creating a two-way relationship between man and the environment derived from respectful modification. It is only in this way that human beings could become a part of it all and that all would become a part of man.

It is not a project that must be studied as an iconic work in the history of architecture, looking only at the built object. Rather, its true value lies in the ability to condense many of the ideas which were found throughout the world, at times in parallel to each other, in a period of such intellectual excitement as the first three decades of the 20th century. It is an exercise of synthesis conceived in a country like Australia, far from the Europe and America, yet which is their neutral workplace in their desire to define their own identity as pioneers in a land still undiscovered.

The process of their personal search was based on a review of all the influences they had received, aware of them yet attempting to create distance in order to experiment with personal alternatives and to be able to define their own language. The new start on a continent with no local references in contemporary architecture and the freedom to reinterpret what had been learned allowed them to discover their true identity. Hoping to turn their ideals into reality, they decided to take action, guided by intuition and reason, and project their work. As mature architects, they made errors and mistakes and even showed ingenuity, but also determination. The coherence of their thought would make up for any shortcomings in their incipient language, aiming to find an alternative to the known and which, contrary to the passive position shown by others, pushed them to turn it all into a built reality. Willing to accept error, they undertook to create a path, though tortuous at times, in an effort to make architecture universal based on the value of the local. Beyond the architecture proposed in the Castlecrag project, a careful look at the figures of Marion Mahony Griffin and Walter Burley Griffin reveals an attitude, a form of activism that is coherent with certain ideals that sought to improve the world in which they lived.

1. JOHNSON, Donald Leslie. Australian Architecture 1901-51: Sources of Modernism. Sydney: University of Sydney, 1980, pp. 63-65.

2. EMERSON, Ralph Waldo. Nature. Boston: James Munroe and Company, 1836, pp. 15-16.

3. TAUT, Bruno. Escritos expresionistas 1919-1920. Madrid: El Croquis Editorial, 1997, pp. 256-258.

4. BANERJ, Shiben. "Inhabiting the world: Architecture, Urbanism, and the Global Moral-Politics of Marion Mahony and Walter Burley Griffin”, Director: Arindam Dutta. Doctoral thesis. Massachusetts Institute of Technology MIT, 2015, pp. 158-162.

5. ULARGUI AGURRUZA, Jesús. De Richardson a Sullivan. Un nuevo espacio social. Barcelona: Fundación Caja de Arquitectos, 2007, pp. 35-38.

6. WALKER, Meredith, KABOS, Adrienne, WEIRICK, James. Building for Nature. Walter Burley Griffin and Castlecrag. New South Wales: Walter Burley Griffin Society, 1994, pp. 7-9. 7. BOYD, Robin. Australia's Home: its Origins, Builders and Occupiers. Melbourne: Melbourne University Press, 1987, pp. 135-136.

8. In reference to the definition of Democracy coined by Louis Sullivan, who believed it not only to be a form of government but also a force that empowers each individual to find their true identity even when still part of a community living model. SULLIVAN, Louis Henry. The Public Papers. Ed. Robert Twombly. Chicago: Chicago University Press, 1988, pp. 97-99.

9. GRIFFIN, Marion Mahony. The Magic of America. Chicago: The Art Institute of Chicago, 1949 (2008), pp. 555-560, [consulted: 05-07-2017]. Available at: http://www.artic. edu/magicofamerica/index.html.

10. TURNBULL, Jeffrey. In Second International Congress on Construction History 3, The Architecture of Walter Burley Griffin: Concrete applications. Melbourne: Construction History Society, 2006, pp. 3135-3136, [consulted: 10-08-2017]. Available at: https://www.arct.cam.ac.uk/Downloads/ichs/vol-3-3133-3154-turnbull.pdf

11. Idem, pp. 3142-3143.

12. GRIFFIN, Dustin. The Writings of Walter Burley Griffin. New York: Cambridge University Press, 2008, pp. 282-284.

13. Idem, pp. 277-278. 


\section{Autor imagen y fuente bibliográfica de procedencia}

Información facilitada por los autores de los artículos:

página 18, 1 (Crédito fotográfico gentileza de Federico Cairoli); página 19, 2 y página 20, 3 (Crédito fotográfico José Luis Uribe Ortiz); página 21, 4 (Crédito fotográfico gentileza de Berenice Gómez Crosa), 5 (Crédito boceto detalle constructivo gentileza de Luis Elgué); página 22, 6 (Crédito fotográfico gentileza de Estudio Elgué), 7 (Crédito boceto detalle constructivo gentileza de Lukas Fuster); página 23, 8 y 9 (Crédito fotográfico gentileza de Federico Cairoli), 10 (Crédito boceto detalle constructivo gentileza de José Cubilla); página 24, 11 y 12 (Crédito fotográfico gentileza de Federico Cairoli); página 29, 1 (Biblioteca Nacional de Australia. P490/7. 1918); página 30, 2, página 31, 3 y página 32, 4 (Javier Mosquera González); página 33, 5 (Biblioteca Nacional de Australia. PIC/9929/2029 LOC Cold Store PIC NICH), 6 (Biblioteca Nacional de Australia. PIC/9929/2110); página 34, 7 (Javier Mosquera González); página 35, 8 (Biblioteca Nacional de Australia. PIC/P2145); página 36, 9 (WikimediaCommons. Autor: Martyman); página 38, 10 (Javier Mosquera González); página 45, 1 (Elaboración propia); página 49, 2 (Elaboración propia, basado en información contenida en: MANNICHE, Peter, Living democracy in Denmark: independent farmers, farmer's cooperation, the folk high schools, cooperation in towns, social and cultural activities, social legislation, a Danish village. 2. ed. Copenhagen: G.E.C. Gad Pub., 1970. ISBN 0837139856), 3 (Elaboración propia, basado en la información contenida en: RUONAVAARA, Hannu. Home ownership and the Nordic housing policies in the 'Retrenchment phase'. En: Conference Building on Housing Ownership, Delft 2008; y en: TSENKOVA, Sasha; VESTERGAARD, Hedvig. Social Housing Provision in Copenhagen. Artículo presentado en ENHR 2011, Toulousse, France); página 50, 4 (Elaboración propia, a partir de la documentación original del proyecto aprobada por las autoridades de Copenhague, En base a la documentación cedida por Fællestegnestuen), 5 (Elaboración propia); página 51, 6 y 7 (Documentación cedida por Fællestegnestuen), 8 (HOLMBERG, Hartvig, ed. -indret selv Deres bolig. Copenhague: KAB, 1979, pp. 8-9); página 52, 9 (Documentación cedida por Fællestegnestuen), 10 (HOLMBERG, Hartvig, ed. -indret selv Deres bolig. Copenhague: KAB, 1979, portada y p. 43); página 53, 11 (HOLMBERG, Hartvig, ed. -indret selv Deres bolig. Copenhagen: KAB, 1979, p. 26), 12 (Elaboración propia); página 59, 1 (Parte superior: The American Woman's Home or Principles of Domestic Science Being a Guide to the Formation and Maintenance of Economical Healthful Beautiful and Christian Homes. New York: J. B. Ford and Company, 1869; The New Housekeeping: Efficiency Studies in Home Management. Garden City-New York: Doubleday, Page \& Company, 1913; The Psychology of Management: The Function of the Mind in Determining, Teaching and Installing Methods of Least Waste. New York: Sturgis \& Walton Company, 1914. Parte inferior: Catharine Beecher (Wikipedia Commons), Christine Frederick (Christine Frederick Archive, Schlesinger Library, Radcliffe Institute, Harvard University), Lillian Gilbreth (Lillian Moller Gilbreth Papers, Sophia Smith Collection, Northampton)); página 60, 2 y página 61, 3 (The American Woman's Home or Principles of Domestic Science Being a Guide to the Formation and Maintenance of Economical Healthful Beautiful and Christian Homes. New York: J. B. Ford and Company, 1869, p. 26, 37, 40); página 62, 4 (Christine Frederick Archive, Schlesinger Library, Radcliffe Institute, Harvard University); página 63, 5 (FREDERICK, Christine. The New Housekeeping: Efficiency Studies in Home Management. Garden City-New York: Doubleday, Page \& Company, 1913, p. 53); página 64, 6 (FREDERICK, Christine. Household Engineering: Scientific Management in the Home. Chicago: American School of Home Economics, 1920); página 65, 7 (Kheel Center for Labor-Management Documentation and Archives, Cornell University Library, Management Engineering), 8 (National Museum of American History, Behring Center, Division of Work and Industry Collection); página 66, 9 y 10 (Elaboración realizada por Carmen Espegel); página 67, 11 (Izquierda: BEECHER, Catharine y BEECHER STOWE, Harriet. The American Woman's Home or Principles of Domestic Science Being a Guide to the Formation and Maintenance of Economical Healthful Beautiful and Christian Homes. New York: J. B. Ford and Company, 1869, p. 23. Derecha: Siedlung Römerstad 1927-1928. Das Neue Frankfurt, n.4-5, Abril-Mayo, 1930, p. 76); página 68, 12 (Izquierda: HAYDEN, Dolores. The Grand Domestic Revolution: A History of Feminist Designs for American Homes, Neighborhoods, and Cities. Cambridge (Massachusetts): MIT Press, 1981, p. 30. Derecha: Ernst May und das Neue Frankfurt, 1925-1930. Catálogo de la exposición en el Deutsches Architekturmuseum Frankfurt am Main. Berlin: Wilhelm Ernst \& Sohn Verlag, 1986, p. 153); página 69, 13 (Superior izquierda y derecha: Restitución gráfica realizada por Carmen Espegel. Inferior izquierda: BEECHER, Catharine y BEECHER STOWE, Harriet. The American Woman's Home or Principles of Domestic Science Being a Guide to the Formation and Maintenance of Economical Healthful Beautiful and Christian Homes. New York: J. B. Ford and Company, 1869, pp. 28-30. Inferior derecha: Das Neue Frankfurt, n.6, Junio, 1929, p. 128), 14 (Izquierda: FREDERICK, Christine. Household Engineering: Scientific Management in the Home. Chicago: American School of Home Economics, 1920, p. 32. Derecha: Margarete SchütteLihotzky: Frankfurter Küche, 1926. Colección y Archivo de Margarete Schütte-Lihotzky en la Universidad de Artes Aplicadas de Viena); página 70, 15 (Izquierda: Original films of Frank and Lillian Gilbreth, promovida por Chicago Chapter, Society for the Advancement of Management, 1910-1924. San Francisco: National Film Preservation Foundation. Derecha: Neues Bauen in Frankfurt am Main, dirigida por Paul Wolff. Frankfurt: Wolff-Film, 1928); página 75, 1 (Enrique Jesús Fernández-Vivancos González a partir del plano de Tapiola de Aarne Ervi de 1963); página 76, 2 (Le Carré Bleu. 1960 nº 3); página 77, 3 y 4 (Museum of Finnish Architecture [MFA]); página 78, 5 (Le Carré Bleu.1958 nº 1 pp. 2-3. Fotógrafo: PIETINEN, Otson); página 79, 6 y 7, página 80, 8, 9 y 10 y página 81, 11 y 12 (Museum of Finnish Architecture [MFA]); página 82, 13 (Museum of Finnish Architecture [MFA]. Fotógrafo: LEHTONEN, Kai R); página 83, 14 (Museum of Finnish Architecture [MFA]); página 84, 15 (PIETILÄ, Reima. Noción Imagen Idea. Espoo: Teknillisen Korkeakoulun Ylioppilaskunta. 1975. Lección 5 y Lección 16); página 90, 1 (Natalia Matesanz Ventura); página 93, 2 y 3 (Fotograma del documental dirigido por Christina Holmes. 2015 [en línea] https://www.youtube.com/watch?v=bB-3Bp2pWh8); página 103, 1 (https://lebbeuswoods.files.wordpress.com/2011/06/Iw-mugshot1.jpg [en línea] [consulta: 22-08-2017]. Disponible en: https://lebbeuswoods.wordpress.com/2011/06/29/anti-journey-to-architecture-1/), 2 (https://lebbeuswoods.wordpress. com/2009/10/06/notebook-01-3-the-last/); página 106, 3 (https://lebbeuswoods.files.wordpress.com/2012/01/lbw-411.jpg[en línea] [consulta: 22-08-2017]. Disponible en: https://lebbeuswoods.wordpress.com/2012/01/02/origins/); página 108, 4 (https://lebbeuswoods.wordpress.com/2009/09/27/the-vagrant-light-of-stars/ [consulta: 22-08-2017]), 5 (https://lebbeuswoods.files.wordpress.com/2010/11/dwg-1.jpg [en línea] [consulta: 22-08-2017]. Disponible en: https://lebbeuswoods.wordpress. com/2010/11/02/drawings-stories/); página 109, 6 (https://lebbeuswoods.wordpress.com/2009/08/01/meta-institutes/ [consulta: 22-08-2017]); página 110, 7 (https:// lebbeuswoods.wordpress.com/2009/03/15/notebook-97-3/ [consulta: 22-08-2017]), 8 (https://lebbeuswoods.wordpress.com/2009/12/19/storm-watch/ [consulta: 2208-2017]); página 111, 9 (https://lebbeuswoods.wordpress.com/2009/06/05/architecture-of-energy/ [consulta: 22-08-2017]), 10 (https://lebbeuswoods.wordpress. com/2011/02/15/a-space-of-light-2/ [consulta: 22-08-2017]); página 112, 11 (https://lebbeuswoods.files.wordpress.com/2012/03/Itpav-may-30-3.jpg [en línea] [consulta: 22-08-2017]. Disponible en: https://lebbeuswoods.wordpress.com/2012/03/25/light-pavilion-under-construction/); página 113, 12 (https://lebbeuswoods.files.wordpress. com/2011/01/cdusk1day.jpg [en línea] [consulta: 22-08-2017] Disponible en: https://lebbeuswoods.wordpress.com/2011/02/15/a-space-of-light-2/); página 114, 13 (https://lebbeuswoods.wordpress.com/2009/09/19/line-up/ [consulta: 22-08-2017]), 14 (https://lebbeuswoods.files.wordpress.com/2011/02/int-5-11.jpg [en línea] [consulta: 22-08-2017]. Disponible en: https://lebbeuswoods.wordpress.com/2011/02/15/a-space-of-light-2/), 15 (Fotografía: Shu He. Imagen cedida por Steven Holl Architects) 